\title{
Effects of Variable Viscosity and Thermal Conductivity on the Flow of Dusty Fluid over a Continuously Moving Plate
}

\author{
G.C. Hazarika \\ Department of Mathematics, \\ Dibrugarh University, Assam 786004
}

\author{
Santana Hazarika \\ Department of Mathematics, \\ Dibrugarh University, Assam 786004
}

\begin{abstract}
This paper investigates the effects of variable viscosity and thermal conductivity of flow and heat transfer of dusty fluid over a continuously moving plate with suction or injection. The fluid viscosity and thermal conductivity are assumed to be vary as an inverse linear function of temperature. The flow governing equations are transformed into ordinary differential equations and the resulting coupled non-linear boundary value problem is solved numerically using Runge-Kutta shooting method. The results are represented graphically to illustrate influence of the various physical parameters on the velocity and temperature profiles of both fluid and dust phases. Skin friction and Nusselt number are also coupled and presented in tabular form.
\end{abstract}

\section{Keywords}

Continuously moving plate, variable viscosity and thermal conductivity, dusty fluid, fluid-particle interaction parameter, shooting method.

\section{INTRODUCTION}

There is a wide range of application of heat transfer and the flow of a viscous fluid induced by a continuously moving or stretching surface. Mainly in chemical industry, power and cooling industry for drying, chemical vapour deposition on surfaces and cooling of nuclear reactors etc it plays a prominent role. The rate of stretching is very important as rapid stretching results in sudden solidification, thereby destroying the properties expected for the outcome.

Sakiadis [11] first studied the behaviour of boundary layer flow of viscous fluid over a stretching flow problem in various aspects. Crane [3] examined the steady twodimensional boundary layer flow of an incompressible and viscous fluid caused by a stretching sheet whose velocity varies linearly with the distance from a fixed point. Chakrabarti et al. [2] also studied the flow and heat transfer of an electrically conducting incompressible fluid past a stretching sheet. Grubka et. al. [6] investigated the heat transfer characteristics of a continuous stretching surface with variable temperature.

The study of the two-phase flows, in which solid spherical particles are distributed in a fluid has a great role in the fields of fluidization, combustion, use of dust in gas cooling systems, centrifugal separation of matter from fluid, petroleum industry, and purification of crude oil, electrostatic precipitation, polymer technology, and fluid droplets sprays. Gireesha et. al. [4] obtained the numerical solution for boundary layer flow and heat transfer of a dusty fluid over a stretching sheet with non-uniform heat source/sink and viscous dissipation. Sharma et. al. [12] studied the effects of dusty viscous fluid on MHD free convection flow with heat and mass transfer past a vertical porous plate.

These studies are based on constant physical properties. More accurate prediction for the flow and heat transfer can be determined by variation of these properties with temperature. But temperature dependent physical properties like viscosity of the fluid and thermal conductivity plays a significant role in fluid mechanics. It plays a great role in underground storage system and geothermal energy extraction. Attia [1] studied the effect of variable viscosity on transient flow of a dusty conducting fluid a parallel plates channel with uniform suction and injection. They assumed the viscosity to vary exponentially with temperature. Unsteady flow of a dusty conducting fluid through porous medium between parallel porous plates with temperature dependent viscosity and heat source was investigated by Shalini et.al. [14]. Khound et. al. [8] observed that a significant variation of velocity distribution and temperature distribution take place with the variation of viscosity parameter and thermal conductivity parameter.

The aim of this present paper is to investigate the effects of variable viscosity and thermal conductivity on the flow of dusty fluid past a moving plate. Using similarity transformation the flow governing equations are transformed into ordinary differential equations, before numerically solve by Runge-Kutta shooting method. The fluid viscosity and thermal conductivity are taken as inverse linear functions of temperature. The numerical results showed that the fluid particle interaction parameter, viscosity variation parameter, thermal conductivity parameter have significant influence on the flow and heat transfer.

\section{MATHEMATICAL FORMULATION OF THE PROBLEM}

Consider a two dimensional steady laminar boundary layer flow of an incompressible viscous dusty fluid over a continuously moving plate. The flow is generated by the action of two equal and opposite forces along the $x$-axis and $y$ axis being normal to the flow. The sheet being stretched with the velocity $u_{w}(x)$ along the $x$-axis, keeping the origin fixed in the fluid of ambient temperature $T$ as shown in figure I. 


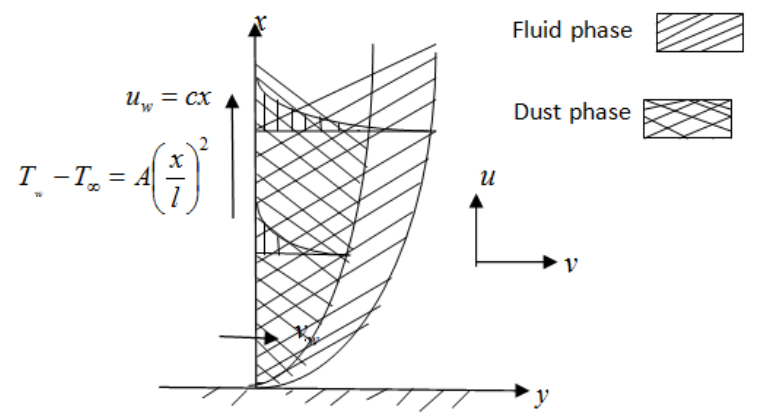

Figure I

Both the fluid and the dust particle clouds are suppose to be static at the beginning. The dust particles are assumed to be spherical in shape and uniform in size and number density of the dust particle is taken as a constant throughout the flow. The fluid properties are assumed to be isotropic and constant except for the fluid viscosity and thermal conductivity.

The flow governing equations for the present problem are:

Equation of continuity for fluid phase

$$
\frac{\partial u}{\partial x}+\frac{\partial v}{\partial y}=0
$$

Equation of momentum for fluid phase

$u \frac{\partial u}{\partial x}+v \frac{\partial u}{\partial y}=\frac{1}{\rho_{\infty}} \frac{\partial}{\partial y}\left(\mu \frac{\partial u}{\partial y}\right)+\frac{K N}{\rho_{\infty}}\left(u_{p}-u\right)$

Equation of energy for fluid phase

$$
\begin{gathered}
\rho_{\infty} c_{p}\left(u \frac{\partial T}{\partial x}+v \frac{\partial T}{\partial y}\right)=\frac{\partial}{\partial y}\left(k \frac{\partial T}{\partial y}\right)+\frac{N c_{p}}{\tau_{T}}\left(T_{p}-T\right) \\
+\frac{N}{\tau_{V}}\left(u_{p}-u\right)^{2}
\end{gathered}
$$

Equation of continuity for dust phase

$$
\frac{\partial}{\partial x}\left(\rho_{p} u_{p}\right)+\frac{\partial}{\partial y}\left(\rho_{p} v_{p}\right)=0
$$

Equation of momentum for dust phase

$$
\begin{aligned}
& u_{p} \frac{\partial u_{p}}{\partial x}+v_{p} \frac{\partial u_{p}}{\partial y}=\frac{K}{m}\left(u-u_{p}\right) \\
& u_{p} \frac{\partial v_{p}}{\partial x}+v_{p} \frac{\partial v_{p}}{\partial y}=\frac{K}{m}\left(v-v_{p}\right)
\end{aligned}
$$

Equation of energy for dust phase $u_{p} \frac{\partial T_{p}}{\partial x}+v_{p} \frac{\partial T_{p}}{\partial y}=-\frac{c_{p}}{c_{m} \tau_{T}}\left(T_{p}-T\right)$

where $(u, v)$ and $\left(u_{p}, v_{p}\right)$ are the velocity components of the fluid and dust particle phases along $x$ and $y$ directions respectively $\mu, \rho_{\infty}, \rho_{p}$ and $N$ are the co-efficient of viscosity of the fluid, density of the fluid, density of the dust phase and number density of the particle phase. $K$ is the stokes' resistance (drag co-efficient), $T$ and $T_{\infty}$ are the fluid temperature within the boundary layer and in the free stream and $m$ is the mass of the dust particle respectively. $T_{p}$ is the temperature of the dust particle, $c_{p}$ and $c_{m}$ are the specific heat of fluid and dust particles, $\tau_{T}$ is the thermal equilibrium time which is the time required by the dust cloud to adjust its temperature to the fluid, $\tau_{V}$ is the relaxation time of the dust particle i.e., the time required by a dust particle to adjust its velocity relative to the fluid and $k$ is the thermal conductivity. In deriving these equations, the drag force is considered for the iteration between the fluid and particle phases.

The boundary conditions for the problem are:

$u=u_{w}(x), v=v_{w}(x), T=T_{w}=T_{\infty}+A\left(\frac{x}{l}\right)^{2}$

at $y=0$,

$u \rightarrow 0, u_{p} \rightarrow 0, v_{p} \rightarrow v, \rho_{p} \rightarrow \omega \rho, T \rightarrow T_{\infty}$,

$T_{p} \rightarrow T_{\infty}$ at $y \rightarrow \infty$

where $u_{w}(x)=c x$ is a stretching velocity, $c>0$ is stretching rate, $\omega$ is the density ratio, $A$ is a positive constant and $l=\sqrt{\frac{v_{\infty}}{c}}$ is a characteristic length.

Lai and Kulacki [9] has assumed the fluid viscosity as

$\frac{1}{\mu}=\frac{1}{\mu_{\infty}}\left[1+\gamma\left(T-T_{\infty}\right)\right]$

or

$\frac{1}{\mu}=a\left(T-T_{r}\right)$

here, $a=\frac{\gamma}{\mu_{\infty}}, T_{r}=T_{\infty}-\frac{1}{\gamma}$

where $\mu$ is the coefficient of dynamic viscosity, $\mu_{\infty}$ is the coefficient of viscosity at free stream, $T$ is the temperature, $T_{\infty}$ is the temperature at free stream, $a$ and $T_{r}$ are constants and their values depend on the reference state and 
thermal property of the fluid. In general $a>0$ for liquids and $a<0$ for gases. $\gamma$ is a constant based on thermal property of the fluid.

Following Khaund and Hazarika [8], has assumed the thermal conductivity as

$\frac{1}{k}=\frac{1}{k_{\infty}}\left[1+\xi\left(T-T_{\infty}\right)\right]$

or, $\frac{1}{k}=c\left(T-T_{k}\right)$

here, $\quad c=\frac{\xi}{k_{\infty}}, T_{k}=T_{\infty}-\frac{1}{\xi}$

where $k$ is the thermal conductivity of the fluid, $k_{\infty}$ is thermal conductivity of the fluid at free stream, $c$ and $T_{k}$ are constants and their values depend on the reference state and thermal property of the fluid. $\xi$ is a constant based on thermal property of the fluid. $c>0$ for liquids and $c<0$ for gases.

Let us introduce the following transformations:

$u=c x f^{\prime}(\eta), v=-\sqrt{v_{\infty} c} f(\eta), \eta=\sqrt{\frac{c}{v_{\infty}}} y$

$u_{p}=c x F(\eta), v_{p}=\sqrt{v_{\infty} c} G(\eta), \rho_{r}=H(\eta)$

$\theta(\eta)=\frac{T-T_{\infty}}{T_{w}-T_{\infty}} \quad \theta_{p}(\eta)=\frac{T_{p}-T_{\infty}}{T_{w}-T_{\infty}}$

where

$$
T-T_{\infty}=A\left(\frac{x}{l}\right)^{2} \theta \quad \text { (Prescribed Power law }
$$

Surface Temperature)

Using the transformations (9-15), in equations (1-7), it is seen that the equation of continuity satisfies identically and rest of the equations becomes:

$f^{\prime \prime \prime}-\frac{\theta^{\prime} f^{\prime \prime}}{\theta-\theta_{r}}-\frac{\theta-\theta_{r}}{\theta_{r}}\left\{f f^{\prime \prime}-f^{\prime 2}+\right.$

$\left.l \beta H\left(F-f^{\prime}\right)\right\}=0$

$$
\begin{aligned}
& \theta^{\prime \prime}-\frac{\theta^{\prime 2}}{\theta-\theta_{k}}-\frac{\left(\theta-\theta_{k}\right) P_{r}}{\theta_{k}}\left\{f \theta^{\prime}-2 f^{\prime} \theta+\right. \\
& \left.\frac{N}{\rho_{\infty} c \tau_{T}}\left(\theta_{p}-\theta\right)+\frac{N E c}{\rho_{\infty} \tau_{V}}\left(F-f^{\prime}\right)^{2}\right\}=0
\end{aligned}
$$

$$
\begin{aligned}
& G F^{\prime}+F^{2}+\beta\left(F-f^{\prime}\right)=0 \\
& G G^{\prime}+\beta(f+G)=0 \\
& H F+H G^{\prime}+G H^{\prime}=0 \\
& 2 F \theta_{p}+G \theta_{p}^{\prime}+\frac{c_{p}}{c c_{m} \tau_{V}}\left(\theta_{p}-\theta\right)=0
\end{aligned}
$$

Here, $l=\frac{m N}{\rho_{p}}, \tau=\frac{m}{k}$ is the relaxation time of the particle phase.

$\beta=\frac{1}{c \tau}$ is the fluid particle interaction parameter.

$\rho_{r}=\frac{\rho_{p}}{\rho}$ is the relative density.

$\operatorname{Pr}=\frac{\mu c_{p}}{k}$ is the Prandtl number.

$E c=\frac{c l^{2}}{A c_{p}}$ is the Eckert number.

$\theta_{r}=\frac{T_{r}-T_{\infty}}{T_{w}-T_{\infty}}=-\frac{1}{\gamma\left(T_{w}-T_{\infty}\right)} \quad$ is the viscosity variation parameter.

$\theta_{k}=\frac{T_{k}-T_{\infty}}{T_{w}-T_{\infty}}=-\frac{1}{\xi\left(T_{w}-T_{\infty}\right)} \quad$ is the thermal conductivity variation parameter.

The transform boundary conditions are

$f=S, f^{\prime}=1, \theta=1$ at $\eta=0$

$f^{\prime}=0, F=0, G=-f, H=\omega, \theta=0, \theta_{p}=0$

as $\eta \rightarrow \infty$

here, $S=-\frac{v_{w}}{\sqrt{v_{\infty} c}}$ is the suction parameter.

The physical quantities of interest are the skin friction coefficient $C_{f}$ and heat transfer coefficient $N u$, the Nusselt number for the surface which are define by

$$
C_{f}=\frac{\tau_{w}}{\rho_{\infty} u_{w}{ }^{2}}=-\frac{\theta_{r}}{\left(\theta-\theta_{r}\right)} \operatorname{Re}^{-\frac{1}{2}} f^{\prime \prime}(0)
$$


where $\tau_{w}=\mu\left(\frac{\partial u}{\partial y}\right)_{y=0}$ is the shear stress at the wall,

and $N u=\frac{x q_{w}}{k_{\infty}\left(T_{w}-T_{\infty}\right)}=-\frac{\theta_{k}}{\theta-\theta_{k}} \operatorname{Re}^{\frac{1}{2}} \theta^{\prime}(0)$

where $q_{w}=k\left(\frac{\partial T}{\partial y}\right)_{y=0}$ is the heat transfer rate at the wall.

\section{RESULTS AND DISCUSSION:}

The system of differential equations (16)- (21) governed by the boundary conditions (22a) and (22b) are solved numerically using Runge-Kutta fourth order method in conjunction with shooting technique. To determine the accuracy of the present method, we should compared the missing values $\mathrm{f}^{\prime \prime}(0)$ and $\theta^{\prime}(0)$ with those values of Salam Odda [13] for various values of $\operatorname{Pr}$ in Table 1 Numerical calculations are carried out for various values of $\theta_{r}, \theta_{k}, \beta$, Ec, Pr.

Velocity profile for various combination of parameters $\operatorname{Pr}=.7$, $\mathrm{Ec}=.05, N=0.5, K=0.2$ are presented in figure 1 to

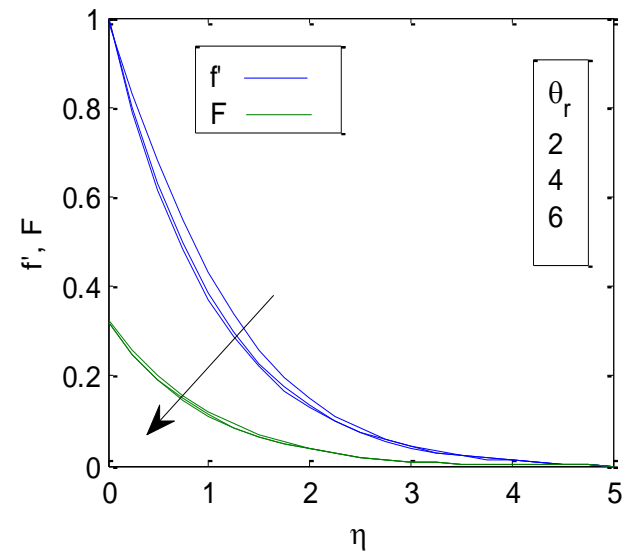

Figure 1: Velocity profile against $\theta_{r}$.

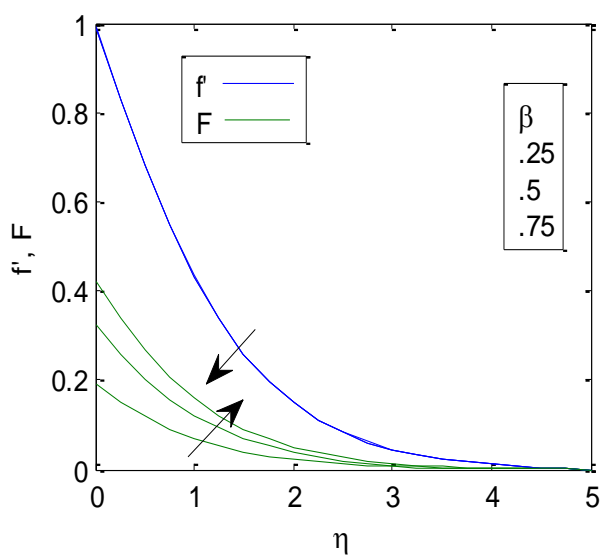

figure 3. It is clearly observed from these figures that the velocity distributions of both fluid phase and dust phase decreases with increase of viscosity variation parameter $\theta_{r}$, thermal conductivity parameter $\theta_{k}$. Also if the fluid particle interaction parameter $\beta$ increases then the fluid phase velocity decrease but dust phase velocity increase.

For numerical calculation we have taken $\tau_{T}=\tau_{V}=0.5, c_{p}=$ $c_{m}=0.2, \rho_{\infty}=0.5$ and $c=1$ unless otherwise stated. From figure 4 to figure 6 it can be concluded that the increases of viscosity variation parameter $\theta_{r}$, thermal conductivity parameter $\theta_{k}$ and the fluid particle interaction parameter $\beta$ retards the temperature of both fluid and dust phases.

The skin-friction coefficient $C_{f}$ which may be taken as the wall shear stress and the heat transfer coefficient i.e. Nusselt number $N u$ are calculated for different values of $\theta_{r}, \theta_{k}$, and $\beta$. From Table 2 and Table 3 it is seen that the skin friction decreases with the increase of $\theta_{r}, \theta_{k}$ but increases with the increase of $\beta$. Nusselt number increases with the increase of $\theta_{k}$ but decreases with the increase of $\theta_{r}$ and $\beta$.

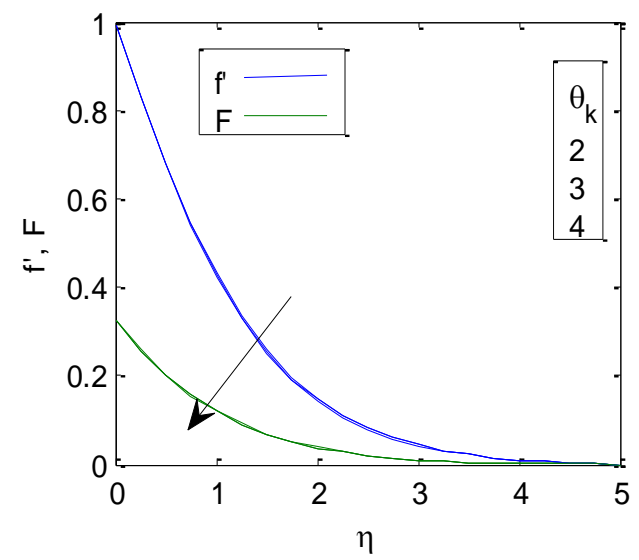

Figure 2: Velocity profile against $\theta_{k}$.

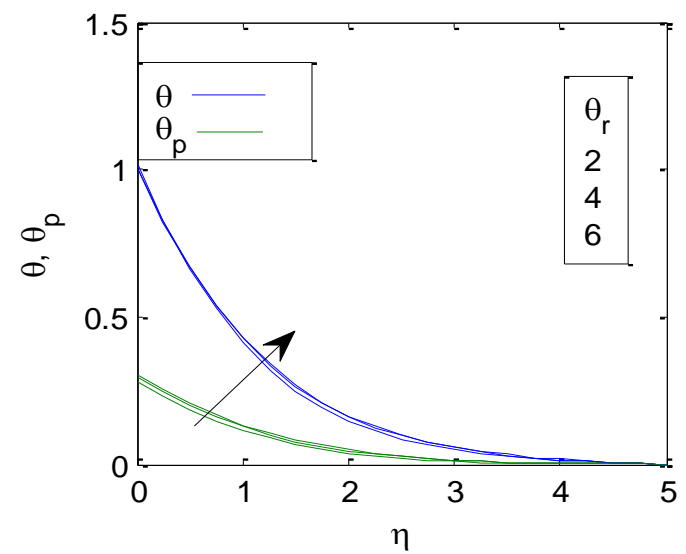


Figure 3: Velocity profile against $\beta$.

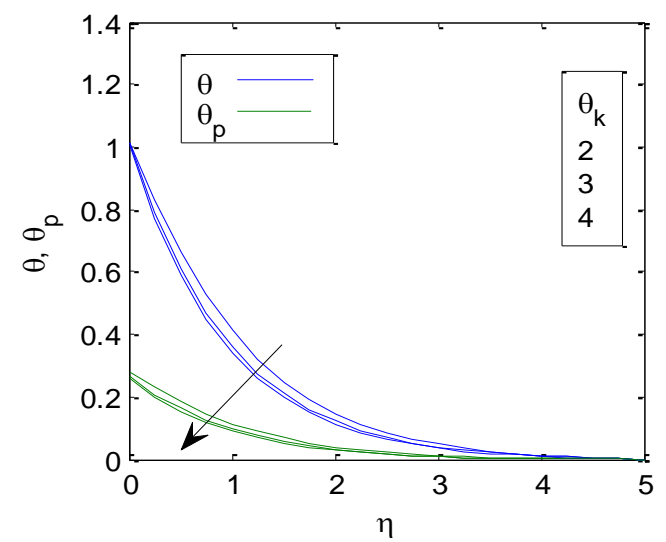

Figure5: Velocity profile against $\theta_{k}$.
Figure 4: Temperature profile against $\theta_{r}$.

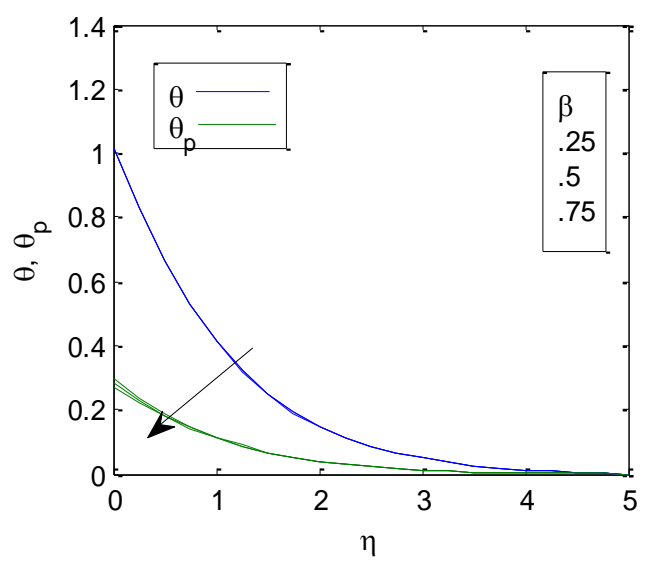

Figure 6: Temperature profile against $\beta$.

Table 1: Comparison of skin friction coefficient $f^{\prime \prime}(0)$ and the heat transfer rate $\theta^{\prime}(0)$ for various values of $\operatorname{Pr}$

Table 1:

\begin{tabular}{|ccccc|}
\hline $\operatorname{Pr}$ & $f^{\prime \prime}(0)$ & $\theta^{\prime}(0)$ & $f^{\prime \prime}(0)$ & $\theta^{\prime}(0)$ \\
\hline \multicolumn{5}{c|}{ dusty } \\
\hline 0.2 & -1.77491 & -0.59624 & -0.0076 & -0.10093 \\
\hline 0.4 & -0.89811 & -0.70919 & -0.00628 & -0.03593 \\
\hline 0.6 & -0.87171 & -0.83648 & -0.00569 & -0.01286 \\
\hline
\end{tabular}

Table 2:

\begin{tabular}{|l|l|l|l|l|l|}
\hline \multicolumn{2}{|c|}{$\theta_{r} f^{\prime \prime}(0)$} & \multicolumn{2}{l}{$\theta^{\prime}(0) \quad-0.09233$} & 0.282226 \\
\hline \multirow{5}{*}{0.2} & 2 & -0.00558 & -0.00792 & -0.092 & \\
\cline { 2 - 6 } & 4 & -0.00578 & -0.00979 & -0.29459 & 0.016137 \\
\cline { 2 - 6 } & 6 & -0.0058 & -0.01048 & -0.65111 & -0.03195 \\
\hline \multirow{5}{*}{0.4} & 2 & -0.00556 & -0.00792 & -0.09179 & 0.279101 \\
\cline { 2 - 6 } & 4 & -0.00575 & -0.00979 & -0.29328 & 0.014812 \\
\cline { 2 - 6 } & 6 & -0.00578 & -0.01048 & -0.64835 & -0.033 \\
\hline \multirow{3}{*}{0.6} & 2 & -0.00554 & -0.00792 & -0.09133 & 0.27661 \\
\cline { 2 - 6 } & 4 & -0.00573 & -0.0098 & -0.29218 & 0.013784 \\
\cline { 2 - 6 } & 6 & -0.00576 & -0.01048 & -0.64605 & -0.03382 \\
\hline
\end{tabular}

Table 3:

\begin{tabular}{|l|ll|l|l|l|}
\hline$\beta$ & $\theta_{k}$ & $f^{\prime \prime}(0)$ & $\theta^{\prime}(0) \quad c f$ & \multicolumn{2}{c|}{$N u$} \\
\hline & 2 & -0.00558 & -0.00792 & -0.09233 & 0.282226 \\
\hline
\end{tabular}




\begin{tabular}{|l|l|l|l|l|l|}
\hline 0.2 & 3 & -0.0055 & -0.00652 & -0.10674 & 0.488425 \\
\cline { 2 - 6 } & 4 & -0.00547 & -0.00599 & -0.11023 & 0.552824 \\
\hline \multirow{4}{*}{0.4} & 2 & -0.00556 & -0.00792 & -0.09179 & 0.279101 \\
\cline { 2 - 6 } & 3 & -0.00548 & -0.00652 & -0.1061 & 0.484706 \\
\cline { 2 - 6 } & 4 & -0.00545 & -0.006 & -0.10957 & 0.548993 \\
\hline \multirow{3}{*}{0.6} & 2 & -0.00554 & -0.00792 & -0.09133 & 0.27661 \\
\cline { 2 - 6 } & 3 & -0.00546 & -0.00652 & -0.10556 & 0.48177 \\
\cline { 2 - 6 } & 4 & -0.00543 & -0.006 & -0.10903 & 0.545988 \\
\hline
\end{tabular}

\section{CONCLUSIONS}

From the above analysis we can conclude that:

1. On decreasing of mass of the dust particles, its relaxation time decreases then the particle interaction parameter increase.

2. Viscosity retards the velocity but enhance the temperature for both fluid and dust phase.

3. Fluid phase temperature is higher than the dust phase temperature as well as the fluid particles temperature is parallel to the dust particles temperature for all the parameters.

4. Velocity and temperature of both fluid and dust phase decreases with thermal conductivity parameter increases.

5. Both the skin friction and Nusselt number decrease with increase of viscosity.

6. The velocity of fluid and dust particles decreases with increase mass concentration of particle because increase in mass concentration of particle reduces mass forces.

\section{ACKNOWLEDGMENTS}

The author is thankful to the unknown reviewers for their valuable suggestions to enhance the quality of the article

\section{REFERENCES}

[1] Attia, H.A., Effect of variable viscosity on transient flow of a dusty conducting fluid in a parallel- plates channel with uniform suction and injection, Tamkang J. Sci. Eng., Vol. 11, no. 2, 2008, pp. 119-126.

[2] Chakrabarti, A. and Gupta, A.S., "Hydromagnetic Flow and Heat Transfer over a Stretching Sheet," Quarterly of Applied Mathematics, Vol. 37, No. 1, 1979, pp. 73-78.

[3] Crane, L. J.. Flow past a stretching sheet, Z. Angew. Math. Phys., 21(1970): 645-647.

[4] Gireesha, B. J., Ramesh, G. K., Abel, M.S. and Bagewadi, C. S., Boundary layer flow and Heat Transfer of a Dusty Fluid Flow over a Stretching Sheet with Nonuniform heat Source/Sink., Int. J. of Multiphase Flow, 37(2011), 977-982.

[5] Gireesha, B.J., Ramesh, G. K., M. and Bagewadi, C. S., Heat Transfer in MHD flow of a dusty fluid over a
Stretching Sheet with viscous dissipation,Adv. In Applied Res., Vol.3, no 4, 2012, pp 2392-2401.

[6] Grubka, L. J. and Bobba, K. M., Heat transfer characteristics of a continuous stretching surface with variable temperature, J. Heat Transfer., 107(1985): 248250 .

[7] Herwig, H., Wickern G., The effects of variable properties on laminar boundary layer flow, Warm and Staffbertr, 20,(1986), pp.47-57.

[8] Khaund, P.K., Hazarika, G.C., The effect of variable viscosity and thermal conductivity on liquid film on an unsteady stretching surface, Proc.of $46^{\text {th }}$ Annual Tech. Session, Ass.Sc.Soc., 2000, pp. 47-56.

[9] Lai, F.C., Kulacki, F.A., The effect of variable viscosity on conductive heat transfer along a vertical surface in a saturated porous medium, Int. J. Heat Mass Transfer, 33, 1990, pp. 1028-1031.

[10] Ling, J.X. and Dybbs, A., Forced convection over a flat plate submerged in a porous medium, variable viscosity case, ASME paper 87-WA/HT- 23 New York, 1987.

[11] Sakiadis, B.C., "Boundary Layer Behaviour on Continuous Solid Surface," AIChE Journal, Vol. 7, No. 1, 1961, pp. 26-28.

[12] Sharma, V.K., Sharma, G., Varshney, N. K., Effects of dusty viscous fluid on MHD free convection flow with heat and mass transfer past a vertical porous plate, Appl. Math. Sci., Vol. 5, no. 77, 2011, pp. 3827-3836.

[13] Salam, A.M., Odda, S.N., Influence of thermal conductivity and variable viscosity on the flow of a micropolar fluid past a continuously moving plate with suction or injection, The Korean Society For Industrial and Applied Mathematics, 2005.

[14] Shalini, Saroa, M. S, Jha, R., Unsteady flow of a dusty conducting fluid through porous medium between parallel porous plates with temperature dependent viscosity and heat source., Int. J. of Scientific Rese. Eng. And Tec., Vol.1, issue 3, 2012, pp. 13-21. 